\title{
transient phenomena in ecology
}

Alan Hastings, ${ }^{1 *}$ Karen C. Abbott, ${ }^{2}$ Kim Cuddington, ${ }^{3}$ Tessa Francis, ${ }^{4}$ Gabriel Gellner, ${ }^{5}$ Ying-Cheng Lai, ${ }^{6}$ Andrew Morozov, ${ }^{7}$ Sergei Petrovskii, ${ }^{7}$ Katherine Scranton, ${ }^{8}$ Mary Lou Zeeman, ${ }^{9}$

${ }^{1}$ Department of Environmental Science and Policy, University of California Davis, CA 95616, USA

${ }^{2}$ Department of Biology, Case Western Reserve University, Cleveland, OH 44106, USA ${ }^{3}$ Department of Biology, University of Waterloo, Waterloo, Ontario, N2L 3G1, Canada ${ }^{4}$ Puget Sound Institute, University of Washington Tacoma, Tacoma, WA 98421, USA

${ }^{5}$ Department of Biology, Colorado State University, Fort Collins, CO 80523, USA

${ }^{6}$ School of Electrical, Computer and Energy Engineering, Arizona State University, Tempe, AZ 85287, USA

${ }^{7}$ Department of Mathematics, University of Leicester, Leicester LE1 7RH, UK

${ }^{8}$ Department of Ecology and Evolutionary Biology, University of California, Los Angeles, CA 90095, USA

${ }^{9}$ Department of Mathematics, Bowdoin College, Brunswick, ME 04011, USA

*To whom correspondence should be addressed; E-mail: amhastings@ucdavis.edu.

The importance of transient dynamics of both ecological systems and the models that describe them has become increasingly recognized. However, previous work has typically treated each instance of these dynamics separately. Here, we review both empirical examples and model systems, and outline a classification of transient dynamics based on ideas and concepts from dynamical systems theory. This classification provides ways to understand the likelihood of transients for particular systems, and to guide investigations to determine 
the timing of sudden switches in dynamics and other characteristics of transients. Implications for both management and underlying ecological theories emerge. 


\section{Introduction}

Understanding ecological dynamics over relevant timescales underpins almost all major questions in ecology, ranging from explanations for observed distributions and abundances of species to population changes through time to management of ecological systems. There is a growing recognition that dynamics on ecological time scales may be different than asymptotic dynamics; these dynamics are called transients. The inherent impermanence of transients means that an ecological system in a transient state can change abruptly, even without any underlying change in environmental conditions (parameters). Conversely, the possibility of long transients implies that an ecological system may remain far from its asymptotic behavior for a long time.

Thus, understanding the implications of transients for ecology depends on understanding potential rapid transitions between two kinds of dynamics, behavior of systems far from their final dynamics, and the underlying time scales for these transitions. However, with the current lack of a systematic framework to facilitate understanding of transient dynamics, each example appears novel and idiosyncratic. Concepts from dynamical systems can provide tools for a more systematic approach to the incorporation of transient dynamics in ecological models and theories, as well as guide applications to natural and managed systems. Tools will emerge for understanding which ecological factors produce long transients, and appropriate responses to the possibility of sudden system changes in management and in experimental and observational studies.

A major ecological question is how to relate observations of changes in dynamics to underlying causes. With transients there may be no underlying proximal cause of a sudden change in dynamics. There may have been no underlying environmental change, or the change may have occurred quite far in the past. In contrast, identification of the proximal factors responsible for regime shifts has been a major focus of attention over the last two decades $(1,2)$. 
One conjectured mechanism is regime shifts occurring as a result of slow, directional, change in ecological parameters, especially when such a change leads to a "bifurcation" of the ecosystem properties (e.g. a disappearance of a stable steady state) $(1,2)$, also known as a "tipping point". In turn, the directional change in parameter values is often assumed to occur due to an exogenous process such as, for instance, global climate change. Intense study of one kind of exogenously-triggered regime shift (those caused by saddle node bifurcations) has provided important insights $(1,3,4)$ across a range of ecological systems. There is, however, a growing body of evidence that we review here, from both empirical and modeling studies, suggesting alternative underlying mechanisms for some regime shifts.

The approach for understanding regime shifts can be extended to a much broader range of phenomena and systems by focusing on transients in ecological systems, where once again ideas from dynamical systems can organize what may at first appear to be a disparate set of observations and explanations. In the cases we focus on here, the ecological dynamics are essentially transient (5-10) and shifts occur in the absence of any clear trend in the environmental properties. Ecological transients can arise for a number of reasons ranging from response to environmental fluctuations to a variety of human interventions. While some transients are short, others can last for a very long time. An ecosystem exhibiting long transient behavior would typically show an apparently stable dynamic (e.g. periodic oscillations, as in Fig. 1a,d,e) over time that may span dozens or even hundreds of generations before experiencing a sudden transition to another state (e.g. extinction) or another regime (e.g. oscillations with a very different mean value). Therefore, long transients may provide an alternative explanation of ecological regimes shifts.

Transients are not an isolated phenomenon, but are related to other aspects of the dynamics of ecological systems that provide challenges for long term prediction. With transient dynamics, the difficulty of predicting the timing of the shift between dynamic behaviors is compounded 
(a)

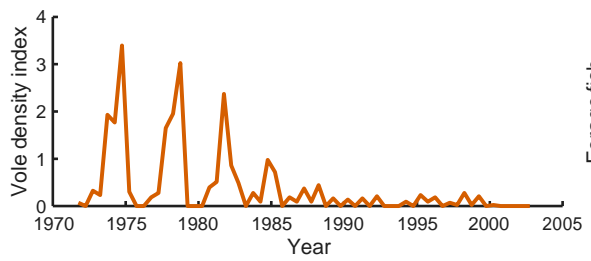

(c)

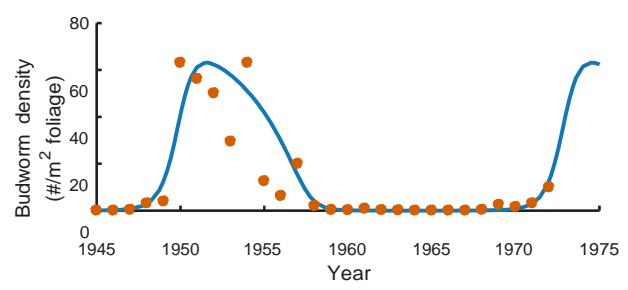

(e)

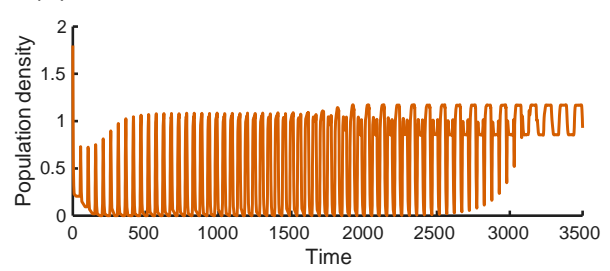

(b)

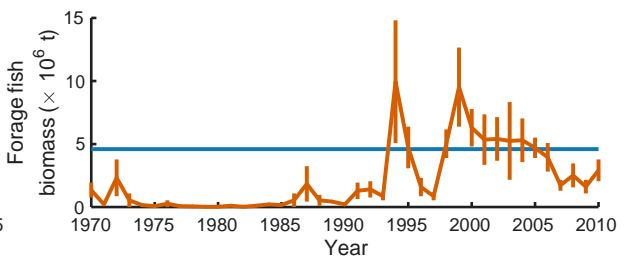

(d)

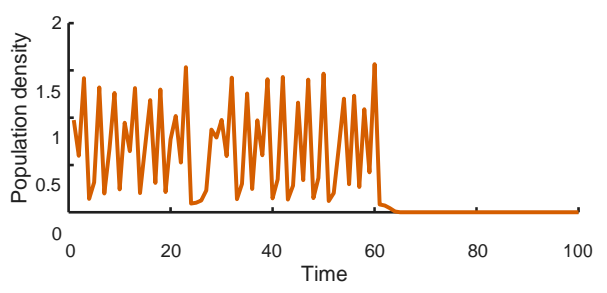

Figure 1: Examples of transient dynamics. (a-c) Empirical examples of regime shifts occurring after long transient dynamics: (a) the population abundance of voles in northern Sweden, showing a transition from large-amplitude periodic oscillations to nearly steady-state dynamics (redrawn from (11)); (b) the biomass of forage fishes in the eastern Scotian Shelf ecosystem; a low-density steady state changes to a dynamical regime with a much higher average density (blue line is the estimated carrying capacity; bars are \pm s.e.m.; redrawn from (12)). (c) Spruce budworm (dots; data from (13)) has a much faster generation time than its host tree, resulting in extended periods of low budworm density interrupted by outbreaks. (c-e) Examples of long transients in population dynamics models: (c) a model (blue line) with fast budworm dynamics and slow foliage dynamics shows qualitative agreement with the data (14); (d) apparently sustainable chaotic oscillation suddenly result in species extinction (15); (e) large-amplitude periodic oscillations that persist over hundreds of generations suddenly transition to oscillations with a much smaller amplitude and a very different mean (16). 
by the difficulty of determining asymptotic behavior from observations of short term behavior (or the converse). Chaotic dynamics limit the time over which accurate predictions are possible (17-19). The permanent influence of external and internal noise on population dynamics also substantially reduces ecological predictability in a number of ways $(20,21)$. Ecological predictions are further complicated by regime shifts $(3,4)$ that occur as underlying environmental conditions slowly change. As a result, any conclusions or estimates made based on the observations before the regime shift simply become irrelevant after the shift. Regime shifts can often result in catastrophic changes in the ecosystem structure and function, in particular leading to species extinction and biodiversity loss.

Although long transients are often observed in ecological data (see Table 1) and have been seen in many different models in ecology $(5,15,16)$ as well as other natural sciences $(22)$, a systematic consideration of this highly relevant phenomenon has been missing so far. Additionally, there has been some confusion about the relationship between regime shifts and long transients. We begin with an overview of ideas from dynamical systems that show why transients are a universal feature of ecological systems. We propose a simple classification scheme that shows that the mechanisms producing transients can be put into a small number of classes. This classification thus provides a new unified framework for incorporating transients into both interpretations of ecological dynamics and into management responses. Additionally, we provide a road-map for future investigations based on open challenges in the study of transient dynamics.

\section{Classification and mechanisms}

The unifying principle underlying past studies of long transients is a focus on multiple time scales (35). One example is regime shifts where slow parameter changes eventually lead to relatively rapid shifts in the state of an ecosystem: there is a slow and a fast time scale. We 
Table 1: Empirical evidence for long ecological transients.

\begin{tabular}{|c|c|c|}
\hline Population(s) & Observed pattern & $\begin{array}{l}\text { Duration in } \\
\text { generations (years) }\end{array}$ \\
\hline $\begin{array}{l}\text { Laboratory population of beetles } \\
\text { (Tribolium spp.) (23) }\end{array}$ & $\begin{array}{l}\text { Switch from a regime with an almost } \\
\text { constant density to large amplitude } \\
\text { oscillations }\end{array}$ & $\begin{array}{l}15(\sim 1.5 \text { yrs }(70 \\
\text { weeks }))\end{array}$ \\
\hline $\begin{array}{l}\text { Growth of macrophytes in shallow } \\
\text { eutrophic lakes in the Nethelands } \\
\text { (24) }\end{array}$ & $\begin{array}{l}\text { Switch from a } \\
\text { macrophyte-dominated state to a } \\
\text { turbid water state }\end{array}$ & $1-5$ (1-5 yrs) \\
\hline $\begin{array}{l}\text { Population of large-bodied benthic } \\
\text { fishes on the Scotian Shelf of } \\
\text { Canada's east coast (12) }\end{array}$ & $\begin{array}{l}\text { Switch from forage fish (and } \\
\text { macroinvertebrate) dominated state } \\
\text { to benthic fish dominated state }\end{array}$ & 5-8 (20 yrs) \\
\hline $\begin{array}{l}\text { Coral and microalgae in the } \\
\text { Caribbean }(25,26)\end{array}$ & $\begin{array}{l}\text { Shifts from coral to macroalgal } \\
\text { dominance on coral reefs }\end{array}$ & $\begin{array}{l}20-25 \text { (corals); } 50-100 \\
\text { (macroalgae) (10 yrs) }\end{array}$ \\
\hline Voles, grouse in Europe (27) & $\begin{array}{l}\text { Switch between cyclic and } \\
\text { non-cyclic regimes or between cyclic } \\
\text { regimes with different periodicity }\end{array}$ & $\begin{array}{l}60 \text { (voles); } 20-30 \\
(\text { lemmings); } 5 \text { (grouse) } \\
(\sim 30 \text { yrs) }\end{array}$ \\
\hline $\begin{array}{l}\text { Dungeness crab (Cancer magister) } \\
\text { (28) }\end{array}$ & $\begin{array}{l}\text { Large amplitude transient } \\
\text { oscillations with further relaxation to } \\
\text { equilibrium }\end{array}$ & $10-15$ (45 yrs) \\
\hline $\begin{array}{l}\text { Zooplankton-algal interactions in } \\
\text { temperate lakes in Germany ( } 29 \text { ) }\end{array}$ & $\begin{array}{l}\text { Variation of amplitude and period of } \\
\text { predator-prey oscillations across the } \\
\text { season }\end{array}$ & $\begin{array}{l}\text { 80-100 (algae); } 5-8 \\
\text { (zooplankton) (1 yr) }\end{array}$ \\
\hline $\begin{array}{l}\text { Planktonic species in chemostat and } \\
\text { temperate lakes }(30)\end{array}$ & $\begin{array}{l}\text { Long term variation of species } \\
\text { densities with extinction of some }\end{array}$ & $\begin{array}{l}40-100(\sim 0.05-0.15 \\
\text { yrs }(3-8 \text { weeks }))\end{array}$ \\
\hline $\begin{array}{l}\text { Laboratory microbial } \\
\text { communities (31) }\end{array}$ & $\begin{array}{l}\text { Slow switch between alternative } \\
\text { stable states }\end{array}$ & $\begin{array}{l}20-40(0.11-0.21 \text { yrs } \\
(6-12 \text { weeks }))\end{array}$ \\
\hline $\begin{array}{l}\text { Grass community in abandoned } \\
\text { agricultural fields in } \\
\text { Netherlands (32) }\end{array}$ & $\begin{array}{l}\text { Long-term existence of a large } \\
\text { number of alternative transient states }\end{array}$ & 10 (9 yrs) \\
\hline $\begin{array}{l}\text { Extinction debt phenomena as a } \\
\text { consequence of habitat loss (plants, } \\
\text { birds, fish, lichens and others (33)) }\end{array}$ & $\begin{array}{l}\text { Long-term extinction of populations } \\
\text { that occurs either steadily or via } \\
\text { oscillations }\end{array}$ & $\begin{array}{l}20-100 \text { (or more) } \\
\text { (1-100 yrs) }\end{array}$ \\
\hline $\begin{array}{l}\text { Fish and invertebrates in watersheds } \\
\text { in western North Carolina, USA (34) }\end{array}$ & $\begin{array}{l}\text { Influence of past habitat structure on } \\
\text { present biodiversity patterns after } \\
\text { restoration }\end{array}$ & $\begin{array}{l}\text { 10-20 (fish); } 40 \\
\text { (invertebrates) (40 yrs) }\end{array}$ \\
\hline $\begin{array}{l}\text { Modeled spruce budworm outbreaks } \\
\text { in balsam fir forests (14) }\end{array}$ & $\begin{array}{l}\text { Budworm outbreaks driven by slow } \\
\text { changes in condition of fir foliage }\end{array}$ & $\begin{array}{l}5 \text { (refoliation); } 50+ \\
\text { (budworm) (50 yrs) }\end{array}$ \\
\hline
\end{tabular}


extend this view in two critically important ways: we ask about the dynamics on both time scales and we include other ways that transients arise. Beyond this emphasis on multiple scales we emphasize that the ecologically relevant time scales are typically not the asymptotic time scales which have been the focus of many ecological modeling studies and which form the basis of theory on which many empirical studies rest. Neither are very short time scales the appropriate focus.

The tools of dynamical systems provide the means for a systematic approach to long transients. Thus a first step is a very short review of concepts from dynamical systems (35) that underly the more traditional view of ecological systems representing and being represented by the asymptotic behavior of mathematical models. The simplest long term, or asymptotic, behavior would be a stable equilibrium; a slightly more complex possibility would be a stable limit cycle. A cycle or an equilibrium are both examples of invariant sets: if the system is at an equilibrium or on a cycle it will remain there forever in the absence of any perturbation or change in parameter values. There are also more complex invariant sets, including chaotic ones. Under the traditional view of ecological systems, on time scales that are relevant for understanding these systems, the focus should not only be on invariant sets, but limited to the stable invariant sets that are approached through time. A major limitation of this view is that the relevant timescale for important ecological questions may be short enough that the asymptotic behavior is not an appropriate description. We can, however, still use ideas from dynamical systems to understand and classify the behavior of ecological systems on these shorter (but not very short) time scales.

There is a broad range of transient patterns in real ecological systems, likely caused by a range of mechanisms (Table 1 ). We can classify those mechanisms into two general categories: those that occur in the vicinity of an invariant set, and those that do not. Within this broad classification we also can identify properties that make a system particularly prone to long 
transients such as the presence of multiple time scales, high dimensionality and stochasticity.

We call a dynamical regime (e.g. a nearly-constant state or persistent cycles) a long transient if it exhibits the following two properties:

- The dynamical regime persists for a sufficiently long time and is quasi-stable (approached over shorter time scales), rather than actually stable. Thus, if the dynamics are observed for a sufficiently long time (in appropriate time units, e.g. generations of a relevant species), a clearly seen transition eventually occurs to another equilibrium or dynamic regime.

- The transition between the regimes occurs on a time scale much shorter than the time of existence of the quasi-stable regime. In other words, the dynamics both before and after the transition last much longer than the time of transition.

Below, we consider a few simple models that exhibit long transients with somewhat different properties. We use these to formalize our classification and describe the mechanisms underlying the long transients.

\section{Ghosts and Crawl-bys}

One class of long transients arises when a system is near a bifurcation. If we imagine a system's dynamics represented as a ball rolling on an uneven surface, wells correspond to stable equilibria and peaks to unstable ones. If placed into a well, the ball will roll to a stable equilibrium (Fig. 2a). Consider now the situation where the surface is being gradually deformed in such a way that one of the wells becomes more and more shallow. Eventually the system passes the tipping point at which the stable equilibrium at the bottom of this well and the adjacent unstable equilibrium both disappear, and the ball starts rolling down the slope (Fig. 2b). However, how fast it starts moving away or, in other words, for how much time it remains in the vicinity of 
the location where the stable equilibrium was before the bifurcation, depends on the flatness of the slope. The flatter the surface is, the longer the ball stays close to its original location before moving away: the long transient emerges. Although beyond the tipping point the system does not possess an equilibrium at this long-lasting state, for a considerable time its dynamics mimic the dynamics of the system with an attractor here (Fig. 2c-d). We call this situation a ghost attractor (36) or simply a ghost.

The origin of a ghost attractor and an example of the long transients it can cause are shown in Fig. 2a-d. To understand the importance of this effect, imagine, for instance, that 'competitor 1 ' in the Fig. 2b,d is a native species competing with an invader. At the early stages of invasion, we expect the native species to be abundant and the invader rare. From these initial conditions, the system can spend considerable time in this state, even if the ultimate asymptotic result is that the invader excludes the native species (as in Fig. 2b). This occurs because the invaded system has conditions that are close to, but distinct from, those that would have allowed the invader and the native species to coexist (Fig. 2a,c). Correspondingly, if the system is only monitored on an intermediate time scale, this long transient dynamic may give an impression that both species will coexist indefinitely - a conclusion that would obviously be erroneous on a longer time scale (Fig. 2d). 
(a)

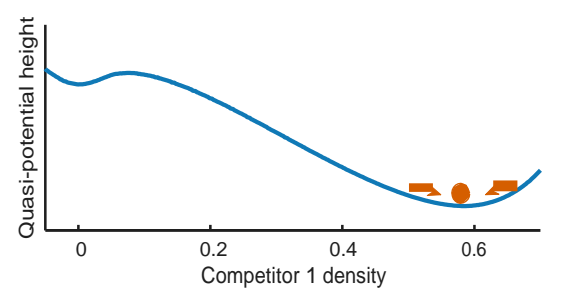

(c)

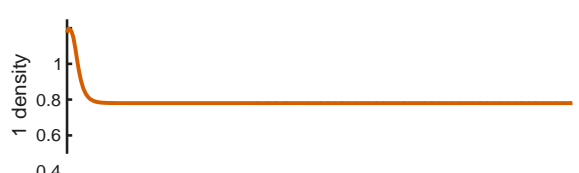

0.4

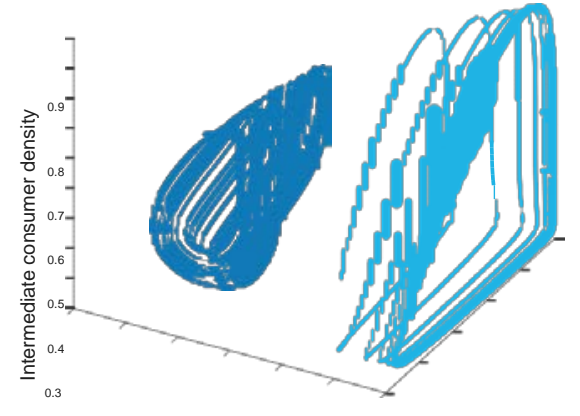

0.2

${ }^{0.1}\left[\begin{array}{c}-- \\ 0.2\end{array}\right.$

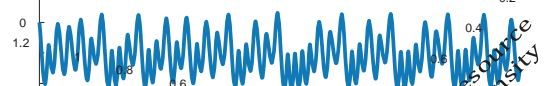

(g) $\begin{aligned} & \text { Predator } \\ & \text { density }\end{aligned}$

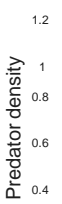

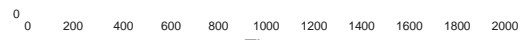

(b)

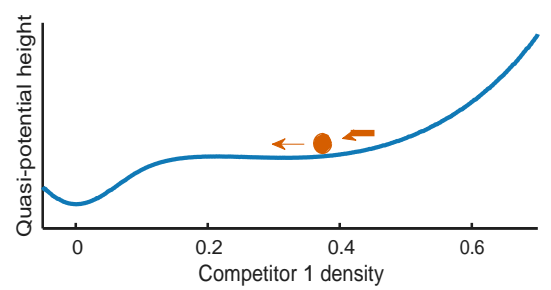

(d)

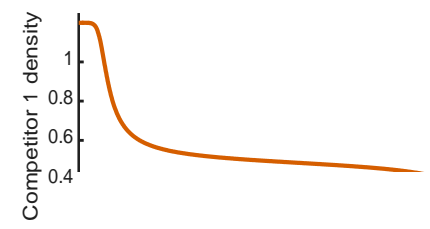

(h)
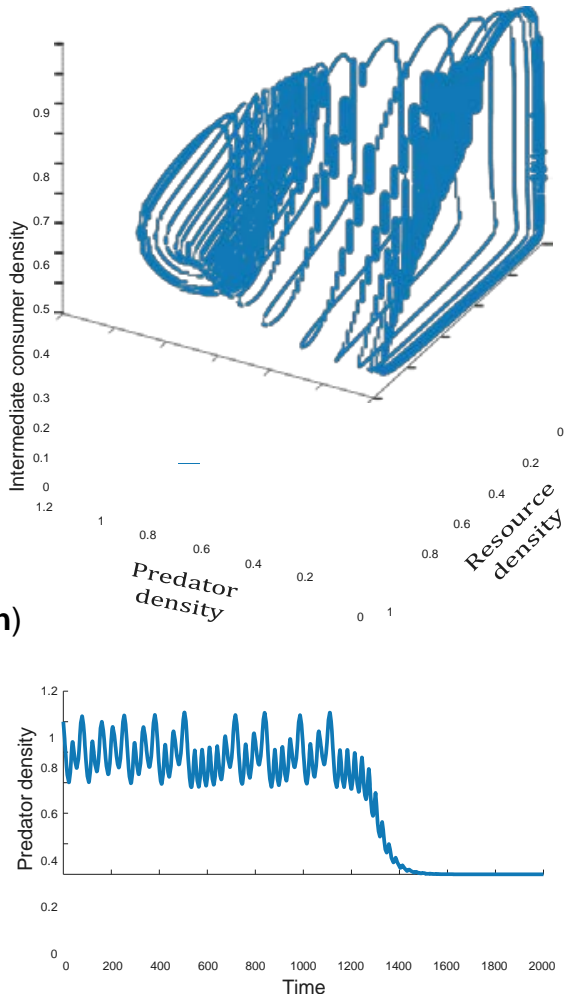
Figure 2: Ghost attractors. Illustration of ghost attractors in (a-d) a 2-species competition model and (e-h) a resource-consumer-predator model. (caption continued on next page) 
Figure 2 caption continued: In the first column (a,c,e,g), there are two stable invariant sets and no ghost attractors. In the second column (b,d,f,h), there is a single stable invariant set, plus a ghost attractor that causes long transients. A bifurcation (tipping point) occurs for parameter values intermediate to these two cases; at this bifurcation, one stable state is lost and a ghost attractor takes its place. $(a, b)$ Dynamics of one of the competitors depicted as a ball on a quasi-potential surface. In (a), a ball to the right of the hump at 0.07 will tend to roll toward the stable equilibrium (well) at 0.58 , as in time series (c). In (b), the surface is relatively flat, rather than containing a well, to the right of $\approx 0.1$; a ball to the right will eventually roll to the stable equilibrium at 0 but will roll very slowly on the flat part of the surface, generating a long transient. There is a ghost attractor at a density around 0.3 , which is visible in time series (d). (e-h) show the same phenomenon with more complex invariant sets: $(e, g)$ The system shows bistability where a chaotic 3-species attractor (dark blue) coexists with a stable consumer-resource limit cycle with no predators (light blue); dark and light trajectories in $(e, g)$ differ only in their initial conditions. $(f, h)$ For parameter values on the other side of a bifurcation that turns the chaotic attractor into a chaotic saddle, any trajectory will eventually converge to the stable limit cycle which is now the global attractor. However, convergence can be slow, as seen in (h), because the chaotic set is now a ghost. Models are: (a-d) 'competitor 1' is $v$ and it competes with species $u$ : $\frac{d u}{d t}=u(1-u)-a_{12} u^{n} v, \frac{d v}{d t}=v\left(v(1-v)-a_{21} u^{n} v\right)$ with $a_{12}=0.9, a_{21}=1.1, y=10$ and $(a, c, e) n=3$; (b,f) $n=1.8$; (d) $n=1.55$; (e-h) from $(37,38)$, where the resource is $R$, consumer $C$, and predator $P$ :

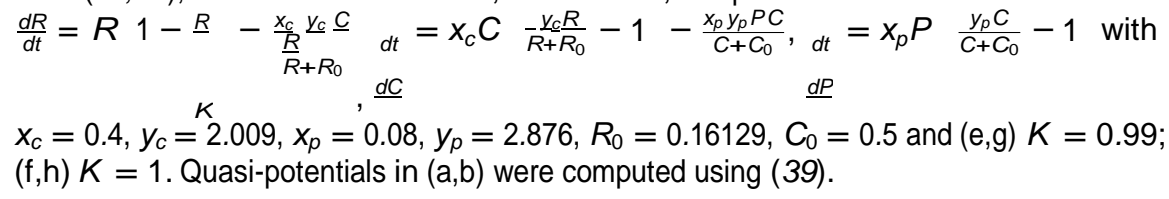

The long transient dynamics in Fig. 2 occur because of a bifurcation that results in the disappearance of a stable equilibrium. Beyond the bifurcation, there is no longer an equilibrium in the vicinity of what is now a ghost, but the system may still spend a long time in this vicinity. In other words, the long transient occurs without an invariant set. In contrast, the second class of transients we define requires the existence of an unstable equilibrium (more specifically, the existence of a saddle). The system approaches the saddle along a stable direction and spends a long time near the saddle. We call this transient a crawl-by. 
(a)

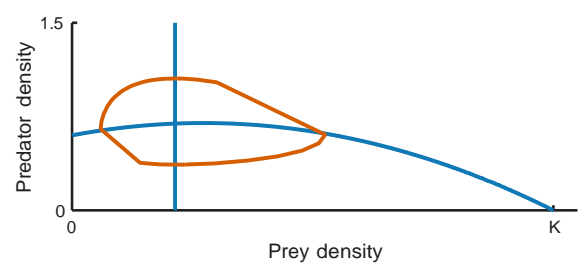

(c)

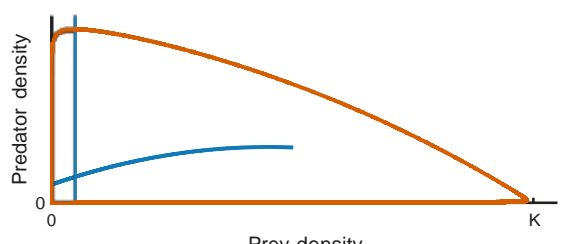

(e)

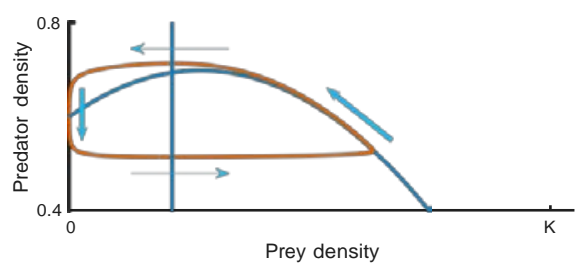

(b)

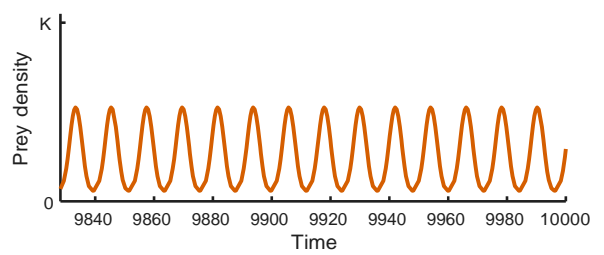

(d)

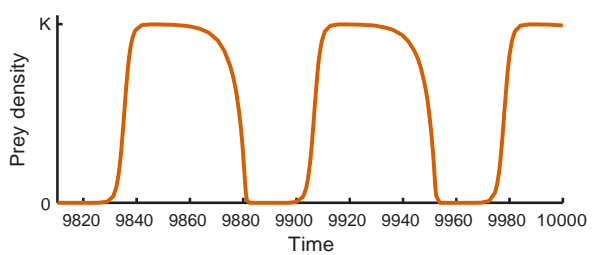

(f)

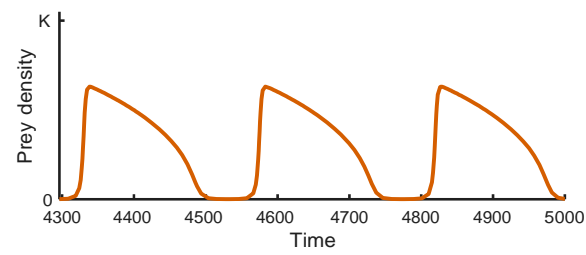

Figure 3: Predator-prey dynamics with and without transients. Predator-prey dynamics $(a, b)$ without long transients, $(c, d)$ with long transients due to crawl-bys, and $(e, f)$ with long transients due to slow-fast dynamics. (a,c,e) The intersection of the predator's and prey's isoclines (blue lines) produces a coexistence equilibrium. When the prey's predator-free carrying capacity, $K$, is beyond a threshold (Hopf bifurcation), the system exhibits limit cycles around this equilibrium. $(a, b)$ For $K$ just beyond this threshold, relatively small limit cycles occur and there are no long transients. (c,d) With an increase in $K$, the cycle grows in size and closely approaches the two saddle points at $(0,0)$ and $(K, 0)$. In (d), crawl-bys are visible at 0 and $K$. (e) When predator (slow) and prey (fast) dynamics occur on very different time scales, the shape of the cycle changes and more horizontal parts of the cycle (thin arrows) proceed much more quickly than more vertical parts (thick arrows). (f) The corresponding time series for the prey shows long transients at 0 and higher prey density. The difference between (f) and (b) is entirely due to the slower predator dynamics in (f). All panels: for prey species $N$ and predator $P, \frac{d N}{d t}=\alpha N \quad 1-\frac{N}{K}-\frac{v N P}{N+h}, \frac{d P}{d t}=E \frac{v v N P}{N+h}-m P$ with $y=2.5, h=1, v=0.5, m=0.4$; 
$(\mathrm{a}, \mathrm{b}, \mathrm{e}, \mathrm{f}) \alpha=1.5, K=2.2 ;$ (c) $\alpha=1.5, K=10 ;$ (d) $\alpha=0.8, K=15 ;(\mathrm{a}-\mathrm{d}) \mathrm{E}=1$; (e-f) $E=0.01$. 
We find examples of this type of long transient in predator-prey systems (see Fig. 3 for details). Note that dynamics with similar properties are observed in more realistic and more complicated models $(23,29)$, and are corroborated by some field and laboratory data $(12,23,29)$ (see also Fig. 1b), which points at the generality of the suggested mechanism.

We note here that crawl-bys and ghosts appear to be similar: having spent some considerable time in the vicinity of its original position, the system (e.g. the ball) eventually moves away. However, a distinction appears when the history of the system is taken into account. For a system to be influenced by a ghost, its initial state must be near the ghost (as in Fig. 2b) or more extreme, such that it passes by the ghost en route to another state (as in Fig. 2d). One reason a system's history might place it near a ghost is if that system recently underwent a change in conditions that caused the ghost attractor to appear. Individual crawl-bys can also occur if the history of the system places it on track to closely approach a saddle, but crawl-bys may also repeat in perpetuity, as in Fig. 3c,d. This occurs because the saddles that give rise to crawlbys are always attracting from some directions while ghosts may or may not have attracting directions.

The mechanisms described above that cause long transients are not restricted to simple dynamics such as steady states or limit cycles. Similar effects can be seen in cases of chaotic dynamics. An illuminating example is given by a resource-consumer-predator system $(37,38)$. In a certain parameter range, this system exhibits chaotic dynamics (see the chaotic attractor (dark blue) in Fig.2e,g). However, a change in parameter values (e.g. an increase in the resource species' carrying capacity) can bring the system to a bifurcation at which the strange attractor disappears (38). Beyond this tipping point, the chaotic dynamic is not sustainable any more; it becomes transient and eventually converges to a stable limit-cycle periodical oscillations (Fig. $2 f, h)$. However, this convergence is slow, so that the dynamics remain essentially chaotic over a long time. Similar dynamics are observed in time-discrete systems (15). This behavior is 
apparently similar to the crawl-by near a saddle point, and indeed the term "chaotic saddle" is used in the physics literature to refer to a non-attracting dynamical invariant set responsible for transient chaos $(40,41)$ (in the dynamical systems theory, it is also called a chaotic supertransient). More generally, a common dynamical mechanism for transient chaos is crises (40), a type of global bifurcation that changes the nature of the underlying chaotic invariant set.

An important point is that one property common for long transients caused by ghost attractors and chaotic crawl-bys is that the system is just beyond the tipping point. So if a parameter controlling a system has pushed the system just past a tipping point there may not be a sudden change, but instead a long transient may result.

\section{Slow-fast systems}

Much of the literature on tipping points considers multiple time scales: fast intrinsic dynamics affected by a slow-changing external factor. However, some systems have multiple time scales within their intrinsic dynamics. This can also lead to transients, as in a prey-predator system written in its general form:

$$
\frac{d N(t)}{d t}=f(N, P, E), \quad \frac{d P(t)}{d t}=E g(N, P, E)
$$

where $E \ll 1$ is a nonnegative dimensionless parameter that quantifies the difference between orders of magnitude for the time scales of prey $(N)$ and predator $(P)(42)$. Such a difference is common in resource-consumer interactions. For example, univoltine insect herbivores that feed on trees have much faster population dynamics than their hosts. Reproduction and mortality rates of zooplankton are typically 10-100 times lower than the corresponding rates of phytoplankton on which the zooplankton feed. Similar differences exist for birds and insects, foxes and voles, etc.

Viewed on the slow time scale, the prey population evolves quickly and is always at its equilibrium with the predator population acting essentially as a slowly changing parameter. 
On the slow time scale the predator population changes with the dynamics determined by the predator equation with the prey assumed to be at an equilibrium. The net result is alternation between long periods of relative stasis and periods of rapid change. An almost steady state dynamic of prey at a very low density accompanied by a gradual decrease in the predator density (as shown by the left-hand side of the cycle in Fig. 3e and each trough in Fig. 3f) can go on for hundreds of generations of prey before suddenly changing to an outbreak in the prey population. The next phase is a slow, gradual decrease in the prey population along with a slow increase in the predator population (the right-hand side of the cycle, and peaks in the prey time series) before accelerating to a fast drop in the prey density. The difference between this dynamic and the transients described in the previous section, in which there is only one intrinsic time scale, is whether the slowly changing variable is viewed as internal or external to the system. This is important, as slowly changing variables are often considered the result of human actions or a changing environment; they could alternatively be viewed as part of the system itself.

\section{Transients in high-dimensional systems}

Most systems outside a laboratory or experimental environment are very high dimensional, due to the presence of space or time delays which greatly increase the potential for transients, including very long transients. In the examples considered so far, all the processes or forces shaping the dynamics are instantaneous and local in space. In real world systems, it is not always so.

Time delays are a common property in ecological dynamics resulting from processes and mechanisms such as nutrient recycling $(43,44)$, maternal effects $(45,46)$, or development in stage-structured populations. Time delays were shown to lead to the emergence of long transients in a few modeling studies $(16,47)$, and there is a certain similarity between delay-caused long transients and those caused by ghost attractors. Systems with time delay are different from 
instantaneous systems not only because of different processes taken into account but also from the viewpoint of dynamical systems theory as the phase space argument and the corresponding analysis become irrelevant. In a general case, even a low-dimensional (e.g. two-species) system with delay is equivalent to an infinite-dimensional instantaneous system $(48,49)$. Altogether, it suggests that time-delay is a separate mechanism that can result in long transient dynamics.

Spatiotemporal dynamical systems are necessarily high-dimensional, and the transient time can increase dramatically with the system size. An early study (5) reported extremely long transients in such systems (Fig. 4a). In systems described by a coupled map lattice, the transient time can increase exponentially with the system size or faster (50), leading to supertransients (51).

\section{Effect of noise}

Until now, we have considered long transient dynamics in deterministic settings, absent noise or stochasticity. In natural systems, noise and random disturbances are inevitable and may create or extend transients.

Noise may affect a system with existing long transient dynamics caused, for example, by a ghost or crawl-by. In transient dynamics caused by a crawl-by, such as the limit cycles of a predator-prey system (Fig. 3c,d), small populations very close to the saddle at $(0,0)$ are vulnerable to stochastic extinctions, where random events may move the system to one of the saddle points, causing either the prey or predator population (or both) to go extinct. Stochasticity in the system near the saddle also has the potential to dramatically alter the length of the transient period, widening the distribution of resulting durations of the transient period or times to convergence (52). Stochasticity in the system near a ghost attractor also widens the distribution of transient periods, depending on the steepness of the surface around the ghost. Noise that is skewed 'uphill' will lengthen the transient, dooming the ball to repeatedly roll nearer the ghost 
(Fig. 2b). Noise can also induce sustained transients or oscillations in a system that - without noise - would exhibit damped oscillations to an equilibrium (53,54, see Fig. $4 \mathrm{~b})$. Noise can also provide a mechanism for transient dynamics of a system to become long lived (Figure 4b). For systems with transient chaos, the interaction with stochasticity can be even more complex (51).

(a)

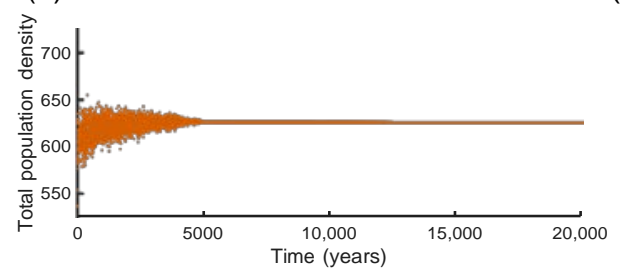

(b)

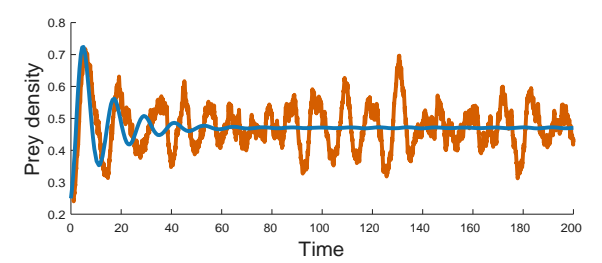

Figure 4: Examples of additional mechanisms leading to long transients. (a) Spatial structure in a simple population model leads to very long transients when the local population growth rate is high (from (5); local dynamics are governed by $N_{t+1}=N_{t} \exp \left(r\left(1-N_{t}\right)\right)$ with $r=3.5$; the total population density summed across all localities is plotted here). (b) For these parameter values ( $\alpha=1.5, K=$ 1.5, $v=2.5, h=1, v=0.5, m=0.4, E=1$ ), the deterministic predator-prey model from Fig. 3 exhibits short transient cycles, then converges to a stable equilibrium point (blue curve). However, when stochasticity is added, the same model will exhibit sustained cycles with approximately the same period (red line; here, stochasticity was incorporated by representing the prey's intrinsic growth rate, $\alpha$, as a random variable with mean 1.5 ). 
Table 2: Overview of long transient (LT) classification and mechanisms.

\begin{tabular}{|c|c|c|c|c|c|}
\hline Type of LT & $\begin{array}{l}\text { Relationship } \\
\text { to invariant } \\
\text { set }\end{array}$ & $\begin{array}{l}\text { Relationship to } \\
\text { bifurcation }\end{array}$ & $\begin{array}{l}\text { Dynamics } \\
\text { mimicked } \\
\text { by LT }\end{array}$ & $\begin{array}{l}\text { Possibility } \\
\text { of recurrent } \\
\text { LTs? }\end{array}$ & $\begin{array}{l}\text { Biological } \\
\text { example }\end{array}$ \\
\hline ghost (Fig. 2) & $\begin{array}{l}\text { no invariant } \\
\text { set }\end{array}$ & $\begin{array}{l}\text { occurs past a } \\
\text { bifurcation where } \\
\text { stable equilibrium } \\
\text { is lost }\end{array}$ & $\begin{array}{l}\text { equilibrium, } \\
\text { cycles, chaos }\end{array}$ & no & $\begin{array}{l}\text { forage } \\
\text { fish (12) } \\
\text { (Fig. 1b) }\end{array}$ \\
\hline $\begin{array}{l}\text { crawl-by } \\
\text { (Fig. 3c,d) }\end{array}$ & $\begin{array}{l}\text { caused by } \\
\text { saddle-type } \\
\text { invariant set }\end{array}$ & none necessary & $\begin{array}{l}\text { equilibrium, } \\
\text { cycles, chaos }\end{array}$ & yes & $\begin{array}{l}\text { phytoplankton- } \\
\text { grazer } \\
\text { models (29) }\end{array}$ \\
\hline $\begin{array}{l}\text { slow-fast } \\
\text { systems } \\
\text { (Fig. 3e,f) }\end{array}$ & $\begin{array}{l}\text { none } \\
\text { necessary }\end{array}$ & $\begin{array}{l}\text { multiple } \\
\text { time-scales }\end{array}$ & $\begin{array}{l}\text { periodic or } \\
\text { aperiodic } \\
\text { cycles }\end{array}$ & $\begin{array}{l}\text { yes, if } \\
\text { invariant } \\
\text { set(s) } \\
\text { present }\end{array}$ & $\begin{array}{l}\text { univoltine } \\
\text { insects (14) } \\
\text { (Fig. 1c) }\end{array}$ \\
\hline $\begin{array}{l}\text { high } \\
\text { dimension } \\
\text { (e.g. time } \\
\text { delays, space } \\
\text { Fig. 4a) }\end{array}$ & $\begin{array}{l}\text { none } \\
\text { necessary }\end{array}$ & none necessary & $\begin{array}{l}\text { equilibrium, } \\
\text { cycles, chaos }\end{array}$ & yes & $\begin{array}{l}\text { chemostat } \\
\text { microbial } \\
\text { communities } \\
(32)\end{array}$ \\
\hline \multirow[t]{2}{*}{$\begin{array}{l}\text { stochasticity } \\
\text { (Fig. 4b) }\end{array}$} & $\begin{array}{l}\text { if invariant set } \\
\text { present: }\end{array}$ & none necessary & $\begin{array}{l}\text { aperiodic } \\
\text { cycles, chaos }\end{array}$ & \multirow[t]{2}{*}{ yes } & \multirow[t]{2}{*}{$\begin{array}{l}\text { Cancer } \\
\text { crabs (28) }\end{array}$} \\
\hline & $\begin{array}{l}\text { if invariant set } \\
\text { absent: }\end{array}$ & $\begin{array}{l}\text { past a bifurcation } \\
\text { where cycles/ } \\
\text { chaos are lost }\end{array}$ & $\begin{array}{l}\text { quasi- } \\
\text { periodic } \\
\text { cycles }\end{array}$ & & \\
\hline
\end{tabular}




\section{Transients in the real world}

The systematic classification of long transient types and mechanisms conducted here provides a framework for recognizing and understanding these dynamics in observed natural systems (Tables 1 and 2). Note that our classification does not include non-autonomous systems, not because long transients do not occur in non-autonomous systems, but because their classification and discussion warrants treatment beyond the scope of the present review. In this section we describe how empirically observed behavior may be the result of long transients in a wide variety of situations and through examples emphasize implications for management.

An empirical example of long transients due to a ghost attractor (similar to that presented in Fig. 2) is the well-documented switch from a macrophyte-dominated state to a turbid water state in freshwater lakes in the Netherlands (24). The study tracks about 70 shallow lakes after a water drawdown that stimulated macrophyte growth, temporarily creating a macrophytedominated, clear water attracting state. When water levels subsequently rose, some of the lakes returned immediately to a turbid state, while others lingered for over 4 years in the clear water state that was no longer stable. In other words, the physical modification to the system caused by the changes in water level resulted in the formation of a clear water ghost attractor that slowed movement towards the turbid water attractor, sometimes quite dramatically. A similar mechanism of long transients due to ghost attractors may underlie the transition from coral to macroalgal dominance reported for Caribbean coral reefs $(25,26)$; the shift from a forage fish state to a state dominated by large-bodied benthic fish species in the Scotian Shelf of Canada's east coast (12) and the shifts between populations of fish and invertebrates in watersheds in western North Carolina after habitat restoration (34).

Long transients due to crawling past a saddle are often observed in planktonic ecosystems, in particular in the interactions between phytoplankton and zooplankton, creating oscillations 
in which periods of high population densities alternate with long periods of low densities (29). Other examples of crawl-bys are given by patterns of cyclic succession reported in a number of ecosystems including competition in communities of sideblotched lizards (55), coral reef invertebrates (56), and heather-moss-bearberry succession (57). In each of these systems, a long dominance of one species is observed before its displacement by the next competitor in the cycle.

Empirical examples of long transients related to slow-fast systems include a number of observations of univoltine insect herbivores that feed on trees (14) (Fig. 1c). At short time scales of a few insect generations the tree density is approximately constant. However, on longer time scales, the impact of the growing insect population may become high enough to cause a suddenly collapse in the quantity or quality of foliage, resulting in a regime shift.

Real ecosystems are often disturbed by noise which can trigger patterns of long transients. A notable example includes the population dynamics of Dungeness crab, Cancer magister, in eight USA west coast ports (28). By combining data analysis with modeling fitted to data it was shown that large amplitude transient oscillations followed by relaxation to an equilibrium occur due to stochastic perturbations of a deterministic system with a stable state. Another example is given by an empirical study on Tribolium (58) in which random perturbations of cyclic population dynamics result in chaotic-like behavior. Seasonal dynamics create a particular structure of environmental stochasticity. For example, in plankton communities in temperate lakes, each cold season "resets" the initial conditions for the warm, growing season. This prevents the system from reaching equilibrium and thereby allows for high biodiversity transients to be the norm $(29,59)$.

High dimensional systems may be likely to possess long transients. For example, slow succession of patterns of biodiversity is found in experimental microbial communities in a chemostat (31). The precise mechanism of these observed long transients is still unclear due to the 
high complexity of systems containing dozens of interacting species and the existence of several time scales. Similarly, long-term existence of a large number of alternative transient states is seen in the restoration of agricultural fields (32), which is also characterized by a high degree of complexity.

\section{Implication for management}

The existence, identification, and forecasting of long transient dynamics in ecosystems have substantial implications for management of ecosystems. Broadly speaking, management is aimed at maintaining or creating a desirable state of the ecosystem. The challenge is in predicting system behavior, given dynamical regime uncertainty. If a system transition is detected, an important question is what has caused it, and how long can it be expected to last? What a study of long transients reveals is that a system may shift in ways that are not simply tracking underlying conditions, so a focus on asymptotic behavior without considering transients may give misleading answers.

In some cases, mechanistic mathematical models that are constructed from first principles, fitted to empirical data, and explored within realistic parameter ranges can help identify whether an ecosystem is currently experiencing transient dynamics. For example, this was done to predict the long transients in the extinction debt of butterflies in the UK (60). In other cases, when it is difficult to distinguish whether observed dynamics are transient or at equilibrium, models of both possibilities can be developed to test the sensitivity of proposed management strategies to the model assumptions.

Incorporating considerations of transient system behavior into management also requires shifting perspectives about the relevant timescale. A fundamental issue is a mismatch between relevant ecological (transient) timescales, and management timescales. Implementation of management plans where long transients are at play will require adjustments to accommodate the 
transient changes in dynamical regime.

Acknowledging transient system behavior affects management assumptions, practices, and interventions. In addition, detecting long transients, and incorporating risk analysis for long transients, requires the development and application of new tools to reflect this change in thinking. A full treatment of the management consequences and opportunities presented by long transients requires further attention beyond the present review-

\section{Implications and Future Directions}

Dramatic changes in ecological dynamics through time represent both great challenges and opportunities for unraveling the forces that regulate ecosystems functioning. Much recent work in this vein has focused on the concept of regime shifts as a rapid response of dynamics to slow changes in environmental conditions (such as climate change, habitat destruction, resource exploitation, etc). However, there are many examples of situations and systems that do not fit into this classical framework; in particular, a shift can suddenly occur in a seemingly constant environment. The existence of long transients explains how it may happen: as we have shown here, the ecosystem dynamics past a tipping point can be very slow, sometimes indistinguishable from the steady state for hundreds of generations (cf. "ghosts"). Long transient dynamics can also be responsible for regime shifts in the absence of any associated tipping point, thus significantly broadening the regime shift paradigm.

The traditional approaches in ecological sciences are usually based on asymptotic dynamics. Here we have shown that this focus is often insufficient and sometimes irrelevant, and needs to be reconsidered in a systematic way. Long transients provide a new dimension in our understanding of observed changes in ecological dynamics. Although the existence of long transients has been previously acknowledged both in theoretical and empirical studies, any systematic approach to this phenomenon has been lacking. We bridge this gap by developing a simple 
classification of different types of long transient dynamics and linking empirical observations to simple prototypical models. As one important result of our investigation, we have arrived at the conclusion that both identifying long transients and understanding their implications (e.g. for ecosystem management) requires coupling across several ecologically relevant time scales.

Identifying from observations whether a natural ecosystem is close to an equilibrium or is experiencing long transient behavior is a major challenge. Perhaps the easiest case is a situation where the population density shows a clear disappearance of periodic cycles of voles, lemmings and grouse in Europe (27) (see Fig. 1a), or the slow steady population decline in the extinction debt phenomena (33). Less evident is the situation where the dynamics do not show a well pronounced trend. In this case, one can compare characteristics of the observed community with those thought to represent equilibrium systems, such as a relatively undisturbed community of a similar type or the same community in the past. These ideas have been implemented to reveal an extinction debt caused by habitat fragmentation, by comparing the current species arearichness relation to historical records (33) and by verifying whether the species-area relation holds (61). As an alternative approach, recently developed techniques make it possible to build an ecological model directly from a time series by reconstructing model equations from data. A particularly promising new approach is based on compressive sensing using a powerful sparse optimization framework (62-65). Once a model is available, its properties can be analyzed, in particular to reveal long transients. However, we argue that any essential progress in this area is only likely to be achieved by combining various methods borrowed from data mining, stochastic modeling and bifurcation theory. Existing methods to identify transients in empirical data are not always reliable and can result in either overlooking the approaching regime shift or in false alarms, which can be very costly. This poses significant new challenges for ecologists, mathematicians and data analysts.

Acknowledging long term transient behavior drastically affects our perception of ecological 
dynamics. First, sudden shifts in dynamics may occur in the absence of underlying parameter changes, i.e. in the absence of the tipping point. Second, analysis of ecological processes must be done across a few relevant timescales rather than focusing only on asymptotic behavior. In particular, one should take into account both fast and slow variables and feedbacks. Third, stochasticity may play a key role in generating long transients, in particular by bringing an ecosystem to the vicinity of an unstable equilibrium (causing a crawl-by) or a ghost. Finally, in the context of ecosystem management practices, it is well known that sometimes long transients may offer a window of response time which would not be available for systems with rapid switches between stable states (66). On the other hand, transients can add more uncertainty to anticipation of regime shifts since such a shift can occur without a noticeable change of parameters.

\section{Acknowledgement}

This work resulted from a working group supported by the National Institute for Mathematical Biological Synthesis, a synthesis center sponsored by the National Science Foundation through award DBI-1300426 with additional support from The University of Tennessee, Knoxville.

\section{References}

1. M. Scheffer, D. Straile, E. H. van Nes, H. Hosper, Limnol. Oceanog. 46, 1780 (2001).

2. S. R. Carpenter, et al., Science 332, 1079 (2011).

3. V. Dakos, et al., PLOS ONE 7, e41010 (2012).

4. C. Boettiger, A. Hastings, J. R. Soc. Interface 9, 2527 (2012).

5. A. Hastings, K. Higgins, Science 263, 1133 (1994). 
6. A. Hastings, Ecol. Lett. 4, 215 (2001).

7. A. Hastings, Trends Ecol. Evol. 19, 39 (2004).

8. A. Hastings, Ecology 91, 3471 (2010).

9. R. S. Wilson, et al., Conse. Biol. 30, 42 (2015).

10. A. Hastings, Proc. Nat. Acad. Sci. USA 113, 14568 (2016).

11. B. Hörnfeldt, Oikos 107, 376 (2004).

12. K. Frank, B. Petrie, J. Fisher, W. Leggett, Nature 477, 8689 (2011).

13. NERC Centre for Population Biology, Imperial College, The Global Population Dynamics Database (1999).

14. D. Ludwig, D. D. Jones, C. S. Holling, J. Ani. Ecol. pp. 315-332 (1978).

15. S. J. Schreiber, Theo. Popu. Biol. 64, 201 (2003).

16. A. Morozov, M. Banerjee, S. Petrovskii, J. Theo. Biol. 396, 116 (2016).

17. R. M. May, Nature 261, 459 (1976).

18. A. Hastings, C. L. Hom, S. Ellner, P. Turchin, H. C. J. Godfray, Ann. Rev. Ecol. System. 24, 1 (1993).

19. J. M. Cushing, R. F. Costantino, B. Dennis, R. Desharnais, S. M. Henson, Chaos in Ecology: Experimental Nonlinear Dynamics, vol. 1 (Elsevier, 2002).

20. J. Greenman, T. Benton, Ame. Natural. 161, 225 (2003).

21. K. C. Abbott, B. C. Nolting, Ecol. Complex. (2016). 
22. Y.-C. Lai, R. L. Winslow, Phys. Rev. Lett. 74, 5208 (1995).

23. J. Cushing, B. Dennis, R. Desharnais, R. Costantino, J. Animal Ecol. 67, 298 (1998).

24. G. V. Geest, H. Coops, M. Scheffer, E. van Nes, Ecosys. 10, 36 (2005).

25. J. Bruno, H. S. W. Precht, E. Selig, V. Schutte, Ecology 90, 14781484 (2009).

26. T. Gardner, I. Cote, J. Gill, A. Grant, A. Watkinson, Science 301, 958960 (2003).

27. R. Ims, J. Henden, S. Killengreen, Trends Ecol. Evol. 23, 79 (2008).

28. K. Higgins, A. Hastings, J. Sarvela, L. Botsford, Science 276, 14311435 (1997).

29. C. Jager, S. Diehl, C. Matauschek, C. Klausmeier, H. Stibor, Ecology 89, 12721286 (2008).

30. U. Sommer, Limnol. Oceanog. 30, 335346 (1985).

31. T. Fukami, Ecology 85, 32343242 (1985).

32. T. Fukami, T. Bezemer, S. Mortimer, W. V. der Putten, Ecol. Lett. 8, 12831290 (2005).

33. R. Bommarco, et al., Irends Ecol. Evol. 24, 564571 (2009).

34. J. Harding, E. Benfield, P. Bolstad, G. Helfman, E. Jones, Proc. Nat. Acad. Sci. USA 95, 1484314847 (1998).

35. C. Kuehn, Multiple Time Scale Dynamics, vol. 1 (Springer, 2016).

36. E. M. Izhikevich, Dynamical Systems in Neuroscience (MIT press, 2007).

37. A. Hastings, T. Powell, Ecology 72, 896 (1991).

38. K. McCann, P. Yodzis, Ame. Natural. 144, 873 (1994). 
39. C. Moore, C. Stieha, B. Nolting, M. K. Cameron, K. C. Abbott, https://cran.rproject.org/web/packages/QPot/index.html (2015).

40. C. Grebogi, E. Ott, J. Yorke, Physica D 7, 181 (1983).

41. T. Kapitaniak, Y.-C. Lai, C. Grebogi, Phys. Lett. A 259, 445 (1999).

42. S. Rinaldi, M. Scheffer, Ecosys. 3, 507 (2000).

43. T. Daufresne, L. Hedin, Proc. Nat. Acad. Sci. USA 102, 9212 (2005).

44. J. Pastor, R. Walker, S. Larsson, Oikos 112, 698 (2006).

45. L. Ginzburg, D. Taneyhill, J. Anim. Ecol. 63, 79 (1994).

46. P. Turchin, Complex Population Dynamics: a Theoretical/Empirical Synthesis (Princeton University Press, Princeton NJ, 2003).

47. M. Jankovic, S. Petrovskii, Theor. Ecol. 7, 335 (2014).

48. Y. Kuang, Delay Differential Equations with Applications in Population Dynamics (Academic Press, New York, 1993).

49. H. Smith, An Introduction to Delay Differential Equations with Sciences Applications to the Life (Springer, Berlin, 2011).

50. J. R. Crutchfield, K. Kaneko, Phys. Rev. Lett. 60, 2715 (1988).

51. Y.-C. Lai, T. Tél, Transient Chaos: Complex Dynamics on Finite Time Scales, vol. 173 (Springer Science \& Business Media, 2011).

52. A. T. Winfree, J. Theo. Biol. 16, 15 (1967).

53. R. M. Nisbet, W. Gurney, Modelling fluctuating populations (Wiley Interscience, 1982). 
54. M. L. Zeeman, et al., Natural Resource Modeling (in press, 2018).

55. B. Sinervo, C. M. Lively, et al., Nature 380, 240 (1996).

56. L. Buss, J. Jackson, Ame. Natural. 113, 223 (1979).

57. R. Marrs, M. J. Hick, J. Appl. Ecol. 23, 1029 (1986).

58. B. Dennis, R. A. Desharnais, J. Cushing, S. M. Henson, R. Costantino, Oikos 102, 329 (2003).

59. J. Huisman, F. J. Weissing, Nature 402, 407 (1999).

60. C. R. Bulman, et al., Ecol. Appl. 17, 1460 (2007).

61. J. MacHunter, W. Wright, R. Loyn, P. Rayment, Can. J. Forest Res. 36, 2756 (2006).

62. E. Candès, J. Romberg, T. Tao, Comm. Pure Appl. Math. 59, 1207 (2006).

63. E. Candes̀, Proceedings of the International Congress of Mathematicians (Madrid, Spain, 2006), vol. 3, pp. 1433-1452.

64. R. G. Baraniuk, IEEE Signal Process. Mag. 24, 118 (2007).

65. E. Candes̀, M. Wakin, IEEE Signal Process. Mag. 25, 21 (2008).

66. T. Hughes, C. Linares, V. Dakos, I. van de Leemput, E. van Nes, Trends Ecol. Evol. 28, 149 (2013). 